\title{
Transcriptome Analysis of Right Ventricular Outflow Tract Myocardium in Repaired TOF Patients with Pulmonary Regurgitation Revealed the Genetic Features of the Right Ventricle Dysfunction
}

\author{
Qiang Fan \\ West China Hospital of Sichuan University \\ Yunfei Ling \\ West China Hospital of Sichuan University \\ Yabo Wang \\ West China Hospital of Sichuan University \\ Shuhua Luo \\ West China Hospital of Sichuan University \\ Qi An ( $D$ anqi@scu.edu.cn ) \\ West China Hospital of Sichuan University
}

\section{Research Article}

Keywords: Tetralogy of Fallot, Pulmonary regurgitation, Right ventricle dysfunction, Transcriptome

Posted Date: January 25th, 2022

DOI: https://doi.org/10.21203/rs.3.rs-1204966/v1

License: (1) This work is licensed under a Creative Commons Attribution 4.0 International License. Read Full License 


\section{Abstract}

Tetralogy of Fallot (TOF) is the most common cyanotic congenital heart defect. Surgical repair can prolong the survival time of TOF patients, but the right ventricle (RV) function of repaired TOF (rTOF) patients will gradually deteriorate because of the residual pulmonary regurgitation (PR). Although pulmonary valve replacement (PVR) can delay the progression of RV dysfunction, it cannot avoid the ultimate RV failure. We conducted a comparative transcriptome analysis of RV outflow track (RVOT) myocardium specimens collected from 6 rTOF patients with PR to define the genetic features of RV dysfunction in this set of patients. We found significant differential expression of a total of 30 genes among which 15 genes were up-regulated and the other 15 genes were down-regulated. There differentially expressed genes were significantly enriched for the biological pathways 'calcium signaling', 'cAMP signaling', 'Adrenergic signaling in cardiomyocytes' and 'Dopaminergic synapse'. This study presents the first global comparative transcriptome profiling of RV dysfunction using RNA-seq and reveals the different gene expression pattern in rTOF patients with PR. These findings have potential translational value because they identify new candidate prognostic markers and targets for the treatment of RV dysfunction.

\section{Background}

Tetralogy of Fallot (TOF) is the most common cyanotic congenital heart defect. From the genetic perspective, the etiology of TOF is multifactorial. Despite successful surgical repair, many patients have chronic volume loading caused by pulmonary regurgitation (PR) that leading to a $40 \%$ probability of right ventricular (RV) failure by the $3^{\text {rd }}$ decade of life ${ }^{[1,2]}$. In addition, some patients developed RV failure before 30 years of age. Heart failure in repaired TOF (rTOF) is recognized as a significant complication. Its occurrence is strongly associated with adverse outcome. Although pulmonary valve replacement (PVR) can delay and improve RV failure, the optimal timing of PVR is still unknown because of the unnatural history of $\mathrm{rTOF}^{[3]}$. Therefore, it is necessary to better understand the molecular and cellular mechanisms which lead to progressive RV enlargement and dysfunction in rTOF patients.

Messenger RNA (mRNA) are coding RNA is a single-stranded nucleotides that carries genetic information to guide protein synthesis which are transcripted from one strand of DNA. Messenger RNA accounts for only $2 \%-5 \%$ of the total RNA of human cells, but it widest in variety and most active in metabolism. Based on its rapid expression, simple sequence composition, low immunogenicity and flexible modification, great progress has been made in mRNA therapy which has been used in the treatment of rare genetic metabolic diseases ${ }^{[4-6]}$. It was reported that interfering with the mRNA of PCSK9 can reduce the production of PCSK9 in the liver and the level of low-density lipoprotein cholesterol, so as to reduce the risk of coronary heart disease ${ }^{[7]}$. The research on mRNA therapy for heart disease is in progress ${ }^{[8]}$, so mining the mRNA profile of RV in rTOF patients is helpful to find potential therapeutic targets and provide necessary theoretical basis of mRNA therapy for RV dysfunction in rTOFpatients.

We hypothesized that mRNA profiles in RV cardiomyocyte would provide insight into the mechanism of disease progression which could then aid in risk stratification and early prediction of RV failure in rTOF patients. For this purpose, we used RNA-seq to analyze the data pertaining to expression levels and performed 
high-resolution transcriptome analyses of the myocardial tissue of RV. The RNA-seq library was sequenced using the DNBSEQ platform, which uses the DNA nanoball technology.

\section{Materials And Methods}

\section{Study population}

Six rTOF patients (4 males, 2 females) undergoing open heart PVR surgery with CPB at the Department of Cardiovascular Surgery of the Sichuan University West China Hospital were enrolled in the study from December 2019 to January 2020. The rTOF patients were divided into two groups, according to the time interval from initial surgical TOF repair to PVR, PVR earlier (PVRe, n=3) and PVR later (PVRI, n=3). PVRe indicated the time interval less than 15 years, PVRI meant the time interval more than 15 years.

The study and protocol were reviewed and approved by Bioethics Committee of Sichuan University West China Hospital. The study was carried out in accordance with the approved guidelines. Written informed consents have been given from all patients and their legal guardian. All procedures in this study were performed in compliance with Helsinki Declaration and national laws.

\section{Tissue sample collection}

RV myocardial tissue samples were obtained from rTOF patients who underwent PVR. The samples from RV were the redundant myocardial at RVOT. Experimental subject enrollment criteria included: rTOF patients receive open heart PVR procedure for the first time. RV myocardial tissues were harvested from six patients with PR after TOF repair. Immediately following surgical resection the RV myocardial was immersed in liquid nitrogen and then stored at $-80^{\circ} \mathrm{C}$ for RNA extraction.

\section{RNA-seq library preparation and sequencing}

We sequenced 6 samples on DNBSEQ Platform in total and generated about 11.18 GB per sample. Sequence reads by quality filters are analyzed in two ways at the level of tran isomers: HISAT [9] and Bowtie2 ${ }^{[10]}$ followed by genes expression quantification using a software package called RSEM12.

\section{Enrichment analysis of DEGs}

We detect DEGs with DEG-seq as requested between PRVe and PRVI. We perform hierarchical clustering for DEGs using heatmap. With the GO annotation result we perform GO functional enrichment using phyper, a function of R. With the KEGG annotation result we perform pathway functional enrichment using phyper, a function of $R$. The $p$ value $<0.05$ means significant enriched.

\section{Results}

Between December 2019 and January 2021, RVOT myocardium from 6 PR patients were collected and their RNA extractedfor RNA-seq analysis. The experimental flow of RNA sequencing is shown in the Figure 1A. 
Clinical characteristics of the 6 patients are described in Table 1.

In our project, we sequenced 6 samples on DNBSEQ Platform in total and generated about 11.18 GB per sample. The average clean reads ratio is $95.7 \%$. The Q20 (error probability of 0.01 ) of the clean nucleotides of all samples was more than $95.88 \%$, with an average of $96.03 \%$. (Table 2 ).

\section{Differential gene expression profiling in PVRe and PVRI}

Global gene expression levels of PVRe $(n=3)$ and PVRI $(n=3)$ demonstrated significant correlation (Figure 1B). 203562 genes were identified in which 202938 of them are known genes and 624 of them are novel genes. 432117 novel transcripts were identified in which 12,672 of them are previously unknown splicing event for known genes, 4826 of them are novel coding transcripts without any known features, and the remaining 318593 are long noncoding RNA.

\section{Transcriptome differences among PVRe and PVRI}

By analyzing the RVOT myocardium specimen between PVRe and PVRI, 30 genes were differentially expressed and annotated, 15 DEGs (50\% of all DEGs) were up-regulated and 15 DEGs (50\% of all DEGs) were downregulated. Volcano plot is shown in Figure 1C. The DEGs heat map for PVRe and PVRI is shown in Figure 2A. The ten most up- and down-regulated DEGs between PVRe and PVRI are listed in Tables 3.

\section{GO classifications and KEGG pathway identification of DEGs}

With DEGs, we perform GO classification. GO has three ontologies: molecular biological function, cellular component and biological process. For the DEGs, the samples in PVRI when compared with PVRe, generated a total of 30 unigenes that can be classified into 46 categories of the $\mathrm{GO}$ functional group. The GO classification results are shown as Figure 2B. Among the $46 \mathrm{GO}$ classifications, 20 biological processes were significantly enriched and showed in Figure 2C, the specific GO terms and the DEGs which were involved in these biological processes are shown in Table 4. With DEGs, we perform KEGG pathway functional enrichment, the pathway functional enrichment results are shown as Figure 2D.

\section{Discussion}

The present study describes genome-wide differential transcriptome profiling underpinning RV dysfunction in patients of rTOF using RNA-seq. Differential gene expression profiles were observed among rTOF patients, dividing them into two groups: PVRe and PVRI. Comparative functional analysis of the DEGs revealed the presence of shared genes and pathways between the two groups. The data obtained from the study provide new insight into the molecular basis of phenotype heterogeneity seen in RV dysfunction of rTOF patients.

Comprehensive genome-wide screening of the transcriptome has helped in understanding the mechanism of disease differentiation and possible diagnostic and treatment value. Herein we compared the transcriptome profiles among the PVRe and PVRI, this helped us to elucidate the pathology of RV failure. By analyzing the expression profiles of the PVRe and PVRI, we could understand the pathology of RV failure at the genome level, and could identify DEGs which may familiarize us with the mechanism of RV failure of rTOF. 
The comparative analysis of transcriptome profiles among the PVRe and PVRI indicated that the pathology of RV failure is multifactorial. Many fundamental biological processes are involved . In addition, KEGG pathway enrichment analyses revealed some specific pathways, including dopaminergic synapse $(P=0.019)$, adrenergic signaling in cardiomyocytes $(P=0.024)$, cAMP signaling pathway $(P$ value $=0.048)$ and calcium signaling pathway $(\mathrm{P}=0.039)$.

With regard to the calcium signaling pathway, previous studies have reported that calcium $\left(\mathrm{Ca}^{2+}\right)$ is the central element of excitation-contraction coupling in cardiomyocytes, but also impacts diverse signaling cascades and influences the regulation of gene expression, referred to as excitation-transcription coupling, $\mathrm{Ca}^{2+}$ dependent pathways are described that modulate gene expression by signal transduction to transcriptional regulators ${ }^{[11,12]}$. This findings suggesting that $\mathrm{Ca}^{2+}$ signaling pathway is involved in myocardial remodeling which can result in the occurrence of heart failure in rTOF patients ${ }^{[13]}$.

Cyclic adenosine monophosphate (cAMP) is a small, hydrophilic molecule, which is an important intracellular second messenger molecule regulated in many physiological processes. The production of cAMP was regulated by a variety of biological stimulus and CAMP exhibited distinct effects on cardiac function and the development of heart failure. But when the cAMP signaling pathway was inhibited the heart function was protected in rat model of heart failure ${ }^{[14]}$. In this study the CAMP signaling pathway was significantly enriched in both groups which suggested that the activation of cAMP pathway in rTOF patients and that the DEGs involved in CAMP signaling pathway participated in the progression of RV failure.

The adrenergic signaling was enriched in RVOT cardiomyocytes in this subset of rTOF patients. Adrenergic signaling was activated by the $\beta_{1}$ and $\beta_{2}$ adrenergic receptor (AR) which are the main $\beta$-AR subtypes expressed

in the human heart ${ }^{[15,16]}$. Under normal conditions, $\beta$-ARs and their signaling pathways modulate both the rate and force of myocardial contraction and relaxation, allowing individuals to respond appropriately to physiological stress or exercise. However, sustained activation of the $\beta$-AR signaling pathways in chronic heart failure can produce harmful biological effects to heart ${ }^{[17]}$. Furthermore, there are cross-talk between $\beta$ adrenergic signaling pathways and other pathways which may contribute to the progression of chronic heart failure ${ }^{[18]}$. Thus, modulating the $\beta$-AR-mediated signaling pathways have been one of the most crucial targets for heart failure therapy $[15,19]$.

The last but not least, the role of dopaminergic synapses in the pathogenesis of nervous diseases and mental disorder has been deeply studied, but there are few studies about that in heart failure. Dopaminergic synapses were also significantly enriched in RVOT cardiomyocytes by transcriptome analysis. Although there is no research elucidating the relationship between dopaminergic synapses and heart failure, our research may provide new clues for the study of the mechanism of RV dysfunction in rTOF patients.

\section{Conclusion}

This study presents the first global comparative transcriptome profiling of RV dysfunction in rTOF patients using RNA-seq and demonstrates the different gene expression patterns of PVRe and PVRI. The coding genes regulating calcium signaling pathway were down-regulated in PVRI, while the coding genes regulating CAMP, adrenergic and dopaminergic synapse signaling pathway were up-regulated in PVRI. This Transcriptome study 
shows that the coding genes regulating calcium signaling pathway may play a role in facilitating the RV dysfunction. Understanding of genetic pathways leading to RV dysfunction is important, as it is a precursor of overt clinical RV dysfunction and will facilitate the development of new therapies that halt progressive RV dysfunction. This may provide a new target for the treatment of RV dysfunction in rTOF patients.

\section{Abbreviations}

TOF: tetralogy of Fallot

rTOF: repaired tetralogy of Fallot

RV: right ventricle

RVOT: right ventricle outflow track

PVR: pulmonary valve replacement

PVRe: PVR earlier, means that the interval from the initial TOF repair to the PVR procedure is less than 15 years.

PVRI: PVR later, means that the interval from the initial TOF repair to the PVR procedure is more than 15 years.

CPB: cardiopulmonary bypass

DEGs: differentially expressed genes

mRNA: messenger RNA

GO: Gene Ontology

KEGG: Kyoto Encyclopedia of Genes and Genomes

FC: fold change

\section{Declarations}

Acknowledgements Not applicable.

Authors' contributions Qiang Fan performed bioinformatics analysis of the data, acquired the results, drafted the manuscript and prepared the figures. Yunfei Ling conceived and designed the study. Yabo Wang, Shuhua Luo and Qi An diagnosed patients, performed the operation, and collected RVOT myocardium specimens. Qi An provided financial support. All authors read and approved the final manuscript.

Funding This study was funded by 1.3.5 project for disciplines of excellence-Clinical Research Incubation Project, West China Hospital, Sichuan University (2018HXFH021) and 1.3.5 project for disciplines of excellence-Clinical Research Incubation Project, West China Hospital, Sichuan University (ZYJC21064). 
Availability of data and materials The datasets generated and analyzed during the current study are available in the SRA public repository at NCBI (https://www.ncbi.nlm.nih.gov/Traces/study/?acc=PRJNA750316) and can be accessed to through SRA Series accession number PRJNA750316.

Ethics approval and consent to participate The study and protocol were reviewed and approved by Bioethics Committee of Sichuan University West China Hospital. The study was carried out in accordance with the approved guidelines. Written informed consents have been given from all patients and their legal guardian. All procedures in this study were performed in compliance with Helsinki Declaration and national laws.

Consent for publication Not applicable.

Competing interests The authors declared that they have no competing interests.

\section{References}

[1] Weldy C S, Syed S A, Amsallem M, et al. Circulating whole genome miRNA expression corresponds to progressive right ventricle enlargement and systolic dysfunction in adults with tetralogy of Fallot[J]. PLoS One, 2020,15(11):e241476.

[2] Mueller A S, McDonald D M, Singh H S, et al. Heart failure in adult congenital heart disease: tetralogy of Fallot[J]. Heart Failure Reviews, 2020,25(4):583-598.

[3] Villafane J, Feinstein J A, Jenkins K J, et al. Hot topics in tetralogy of Fallot[J]. J Am Coll Cardiol, 2013,62(23):2155-2166.

[4] Weng Y, Li C, Yang T, et al. The challenge and prospect of mRNA therapeutics landscape[J]. Biotechnology advances, 2020,40:107534.

[5] Martini P G V, Guey L T. A New Era for Rare Genetic Diseases: Messenger RNA Therapy[J]. Human Gene Therapy, 2019,30(10):1180-1189.

[6] Lei S, Zhang X, Li J, et al. Current Progress in Messenger RNA-Based Gene Therapy[J]. J Biomed Nanotechnol, 2020,16(7):1018-1044.

[7] Preiss D, Mafham M. PCSK9 inhibition: the dawn of a new age in cholesterol lowering?[J]. Diabetologia, 2017,60(3):381-389.

[8] Magadum A, Kaur K, Zangi L. mRNA-Based Protein Replacement Therapy for the Heart[J]. Mol Ther, 2019,27(4):785-793.

[9] Kim D, Langmead B, Salzberg S L. HISAT: a fast spliced aligner with low memory requirements[J]. Nature methods, 2015,12(4):357-360.

[10] Langmead B, Trapnell C, Pop M, et al. Ultrafast and memory-efficient alignment of short DNA sequences to the human genome[J]. Genome biology, 2009,10(3):R25. 
[11] Dewenter M, von der Lieth A, Katus H A, et al. Calcium Signaling and Transcriptional Regulation in Cardiomyocytes[J]. Circulation research, 2017,121(8):1000-1020.

[12] Gilbert G, Demydenko K, Dries E, et al. Calcium Signaling in Cardiomyocyte Function[J]. Cold Spring Harbor perspectives in biology, 2020,12(3):a35428.

[13] Cartwright E J, Mohamed T, Oceandy D, et al. Calcium signaling dysfunction in heart disease[J]. BioFactors (Oxford), 2011,37(3):175-181.

[14] Lin B, Feng D G, Xu J. microRNA-665 silencing improves cardiac function in rats with heart failure through activation of the cAMP signaling pathway[J]. Journal of cellular physiology, 2019,234(8):13169-13181.

[15] Lohse M J, Engelhardt S, Eschenhagen T. What is the role of beta-adrenergic signaling in heart failure?[J]. Circ Res, 2003,93(10):896-906.

[16] Wallukat G. The beta-adrenergic receptors[J]. Herz, 2002,27(7):683-690.

[17] Port J D, Bristow M R. Altered Beta-adrenergic Receptor Gene Regulation and Signaling in Chronic Heart Failure[J]. Journal of molecular and cellular cardiology, 2001,33(5):887-905.

[18] Fujita T, Ishikawa Y. Apoptosis in Heart Failure: The Role of the $\beta$-Adrenergic Receptor-Mediated Signaling Pathway and p53-Mediated Signaling Pathway in the Apoptosis of Cardiomyocytes[J]. Circulation journal : official journal of the Japanese Circulation Society, 2011,75(8):1811-1818.

[19] Schumacher-Bass S M, Traynham C J, Koch W J. G protein-coupled receptor kinase 2 as a therapeutic target for heart failure[J]. Drug Discovery Today: Therapeutic Strategies, 2012,9(4):e155-e162.

\section{Tables}

Table 1. Characteristics of patients in RNA-sequencing cohort

\begin{tabular}{|c|c|c|c|c|c|c|c|c|}
\hline \multirow[b]{2}{*}{ Group } & \multirow{2}{*}{$\begin{array}{l}\text { Patient } \\
\text { number }\end{array}$} & \multirow[t]{2}{*}{ Sex } & \multirow{2}{*}{$\begin{array}{l}\text { Age } \\
\text { at } \\
\text { TOF } \\
\text { repair } \\
\text { (y) }\end{array}$} & \multirow{2}{*}{$\begin{array}{l}\text { Age } \\
\text { at } \\
\text { PVR } \\
\text { (y) }\end{array}$} & \multicolumn{4}{|c|}{ Parameters of CMR at PVR } \\
\hline & & & & & LVEF(\%) & $\operatorname{RVEDVi}\left(\mathrm{ml} / \mathrm{m}^{2}\right)$ & $\operatorname{RVESVi}\left(\mathrm{ml} / \mathrm{m}^{2}\right)$ & $\operatorname{RVEF}(\%)$ \\
\hline \multirow[t]{3}{*}{ PVRI } & 1 & Male & 11 & 29 & 48.0 & 167.1 & 91.2 & 45.6 \\
\hline & 2 & Male & 11 & 29 & 55.5 & 212.9 & 118.4 & 44.4 \\
\hline & 3 & Female & 5 & 25 & 38.7 & 159.7 & 86.7 & 45.7 \\
\hline \multirow[t]{3}{*}{ PVRe } & 4 & Female & 7 & 22 & 49.9 & 128.8 & 68.6 & 46.7 \\
\hline & 5 & Male & 1 & 10 & 69.3 & 133.8 & 62.2 & 53.6 \\
\hline & 6 & Male & 8 & 14 & 48.7 & 167.6 & 109.1 & 34.3 \\
\hline
\end{tabular}

Table 2. Summary of sequencing data for each sample. 


\begin{tabular}{|lllllll|}
\hline & $\begin{array}{l}\text { Total Raw } \\
\text { Reads }(\mathrm{M})\end{array}$ & $\begin{array}{l}\text { Total Clean } \\
\text { Reads (M) }\end{array}$ & $\begin{array}{l}\text { Total Clean } \\
\text { Bases (Gb) }\end{array}$ & $\begin{array}{l}\text { Clean Reads } \\
\text { Q20 }(\%)\end{array}$ & $\begin{array}{l}\text { Clean Reads } \\
\text { Q30 }(\%)\end{array}$ & $\begin{array}{l}\text { Clean Reads } \\
\text { Ratio }(\%)\end{array}$ \\
\hline PVRe1 & 78.88 & 75.04 & 11.26 & 95.89 & 90.35 & 95.14 \\
\hline PVRe2 & 78.88 & 74.99 & 11.25 & 96.07 & 90.74 & 95.07 \\
\hline PVRe3 & 78.88 & 74.83 & 11.22 & 96.23 & 91.07 & 94.86 \\
\hline PVRI1 & 77.13 & 74.15 & 11.12 & 96.03 & 90.69 & 96.14 \\
\hline PVRI2 & 75.37 & 73.12 & 10.97 & 95.89 & 90.39 & 97.01 \\
\hline PVRI3 & 78.88 & 74.85 & 11.23 & 96.21 & 91.02 & 94.89 \\
\hline
\end{tabular}

Clean Reads Ratio $(\%)=$ Total Clean Reads/Total Raw Reads

Table 3. Top ten up-and down-regulated DEGs in PVRe vs. PVRI based on statistical significance 


\begin{tabular}{|c|c|c|c|c|c|c|c|}
\hline \multicolumn{4}{|c|}{ Up regulated genes } & \multicolumn{4}{|c|}{ Down regulated genes } \\
\hline $\begin{array}{l}\text { Gene } \\
\text { symbol }\end{array}$ & Gene annotation & Log2FC & $\begin{array}{l}\mathrm{P} \\
\text { value }\end{array}$ & $\begin{array}{l}\text { Gene } \\
\text { symbol }\end{array}$ & $\begin{array}{l}\text { Gene } \\
\text { annotation }\end{array}$ & & $\begin{array}{l}P \\
\text { value }\end{array}$ \\
\hline CTAG2 & $\begin{array}{l}\text { cancer/testis } \\
\text { antigen } 2\end{array}$ & 20.03 & $3 e-7$ & H2AC19 & $\begin{array}{l}\text { H2A clustered } \\
\text { histone } 19\end{array}$ & 22.01 & $2 e-8$ \\
\hline SFRP5 & $\begin{array}{l}\text { secreted frizzled } \\
\text { related protein } 5\end{array}$ & 3.71 & $4 e-7$ & GSTM1 & $\begin{array}{l}\text { glutathione S- } \\
\text { transferase } \\
\text { mu } 1\end{array}$ & 7.70 & $\begin{array}{l}8.46 \mathrm{e}- \\
6\end{array}$ \\
\hline GRIA3 & $\begin{array}{l}\text { glutamate } \\
\text { ionotropic receptor } \\
\text { AMPA type subunit } \\
3\end{array}$ & 3.70 & $\begin{array}{l}7.83 e^{-} \\
5\end{array}$ & KCNQ2 & $\begin{array}{l}\text { potassium } \\
\text { voltage-gated } \\
\text { channel } \\
\text { subfamily Q } \\
\text { member 2 }\end{array}$ & 6.74 & $\begin{array}{l}2.23 e- \\
6\end{array}$ \\
\hline ATP1B4 & $\begin{array}{l}\text { ATPase } \mathrm{Na}+/ \mathrm{K}+ \\
\text { transporting family } \\
\text { member beta } 4\end{array}$ & 2.91 & $\begin{array}{l}2.60 \mathrm{e}- \\
6\end{array}$ & $\mathrm{P} 2 \mathrm{R} X 3$ & $\begin{array}{l}\text { purinergic } \\
\text { receptor P2X } \\
3\end{array}$ & 3.31 & $\begin{array}{l}1.7 e^{-} \\
15\end{array}$ \\
\hline$X G$ & Xg glycoprotein & 2.90 & $\begin{array}{l}4.57 e^{-} \\
5\end{array}$ & LM01 & $\begin{array}{l}\text { LIM domain } \\
\text { only } 1\end{array}$ & 2.41 & $1 e-7$ \\
\hline GLT1D1 & $\begin{array}{l}\text { chromosome } 2 \\
\text { open reading frame } \\
40\end{array}$ & 2.70 & $3 e-8$ & FAM216B & $\begin{array}{l}\text { family with } \\
\text { sequence } \\
\text { similarity } 216 \\
\text { member B }\end{array}$ & 2.34 & $\begin{array}{l}5.39 \mathrm{e}- \\
6\end{array}$ \\
\hline NEB & nebulin & 2.68 & $5 e-7$ & RORB & $\begin{array}{l}\text { RAR related } \\
\text { orphan } \\
\text { receptor B }\end{array}$ & 2.09 & $6 e-7$ \\
\hline APOA1 & apolipoprotein A1 & 2.57 & $\begin{array}{l}2.6 \mathrm{e}- \\
54\end{array}$ & SDK1 & $\begin{array}{l}\text { sidekick cell } \\
\text { adhesion } \\
\text { molecule } 1\end{array}$ & 1.82 & $\begin{array}{l}2.39 \mathrm{e}- \\
6\end{array}$ \\
\hline CHRFAM7A & $\begin{array}{l}\text { CHRNA7 (exons 5- } \\
\text { 10) and FAM7A } \\
\text { (exons A-E) fusion }\end{array}$ & 2.55 & $\begin{array}{l}6.83 e- \\
5\end{array}$ & NINL & ninein like & 1.42 & $5 e-9$ \\
\hline CCN5 & $\begin{array}{l}\text { cellular } \\
\text { communication } \\
\text { network factor } 5\end{array}$ & 1.95 & $\begin{array}{l}4.35 e^{-} \\
5\end{array}$ & RAB11FIP1 & $\begin{array}{l}\text { RAB11 family } \\
\text { interacting } \\
\text { protein } 1\end{array}$ & 1.38 & $3 e-7$ \\
\hline
\end{tabular}

FC: fold change

Table 4. GO biologic processesand the up- and down-regulated genes in PVRe vs. PVRI based on GO terms 


\begin{tabular}{|c|c|c|c|}
\hline & $\begin{array}{l}\text { Up-regulated genes } \\
\text { (gene ID) }\end{array}$ & $\begin{array}{l}\text { Down-regulated } \\
\text { genes (gene ID) }\end{array}$ & Pvalue \\
\hline monovalent inorganic cation transport & ATP1B4(23439) & P2RX3(5024) & $<0.01$ \\
\hline modulation of chemical synaptic transmission & $\begin{array}{l}\text { GRIA3(2892) } \\
\text { LRP8(7804) }\end{array}$ & $\mathrm{P} 2 \mathrm{R} X 3(5024)$ & $<0.01$ \\
\hline \multirow[t]{2}{*}{ retinoid metabolic process } & APOA1(335) & NA & $<0.01$ \\
\hline & LRP8(7804) & & \\
\hline $\begin{array}{l}\text { negative regulation of cytokine secretion } \\
\text { involved in immune response }\end{array}$ & APOA1(335) & NA & $<0.01$ \\
\hline \multirow[t]{3}{*}{ ion transport } & ATP1B4(23439) & P2RX3(5024) & $<0.01$ \\
\hline & GRIA3(2892) & KCNQ2(3785) & \\
\hline & CHRFAM7A(89832) & & \\
\hline $\begin{array}{l}\text { substrate-dependent cell migration, cell } \\
\text { attachment to substrate }\end{array}$ & TNFRSF12A(51330) & NA & $<0.01$ \\
\hline \multirow[t]{2}{*}{ chemical synaptic transmission } & TNFRSF12A(51330) & P2RX3(5024) & $<0.01$ \\
\hline & & KCNQ2(3785) & \\
\hline somatic muscle development & NEB(4703) & & $<0.01$ \\
\hline \multirow[t]{3}{*}{ biological process } & CTAG2(30848) & $\mathrm{H} 2 \mathrm{AC} 19(723790)$ & $<0.01$ \\
\hline & NEB(4703) & RGPD8(727851) & \\
\hline & XG(7499) & & \\
\hline glucocorticoid metabolic process & APOA1(335) & NA & $<0.01$ \\
\hline $\begin{array}{l}\text { negative regulation of very-low-density } \\
\text { lipoprotein particle remodeling }\end{array}$ & APOA1(335) & NA & $<0.01$ \\
\hline urinary bladder smooth muscle contraction & NA & P2RX3(5024) & $<0.01$ \\
\hline protein oxidation & APOA1(335) & & $<0.01$ \\
\hline nitrobenzene metabolic process & NA & GSTM1(2944) & $<0.01$ \\
\hline ammon gyrus development & NA & LRP8(7804) & $<0.01$ \\
\hline regulation of intestinal cholesterol absorption & APOA1(335) & NA & $<0.01$ \\
\hline regulation of actin filament length & NEB(4703) & NA & $<0.01$ \\
\hline $\begin{array}{l}\text { negative regulation of ion transmembrane } \\
\text { transport }\end{array}$ & CHRFAM7A(89832) & NA & $<0.01$ \\
\hline amacrine cell differentiation & NA & $\operatorname{RORB}(6096)$ & $<0.01$ \\
\hline lipoprotein biosynthetic process & APOA1(335) & NA & $<0.01$ \\
\hline
\end{tabular}




\section{Figures}
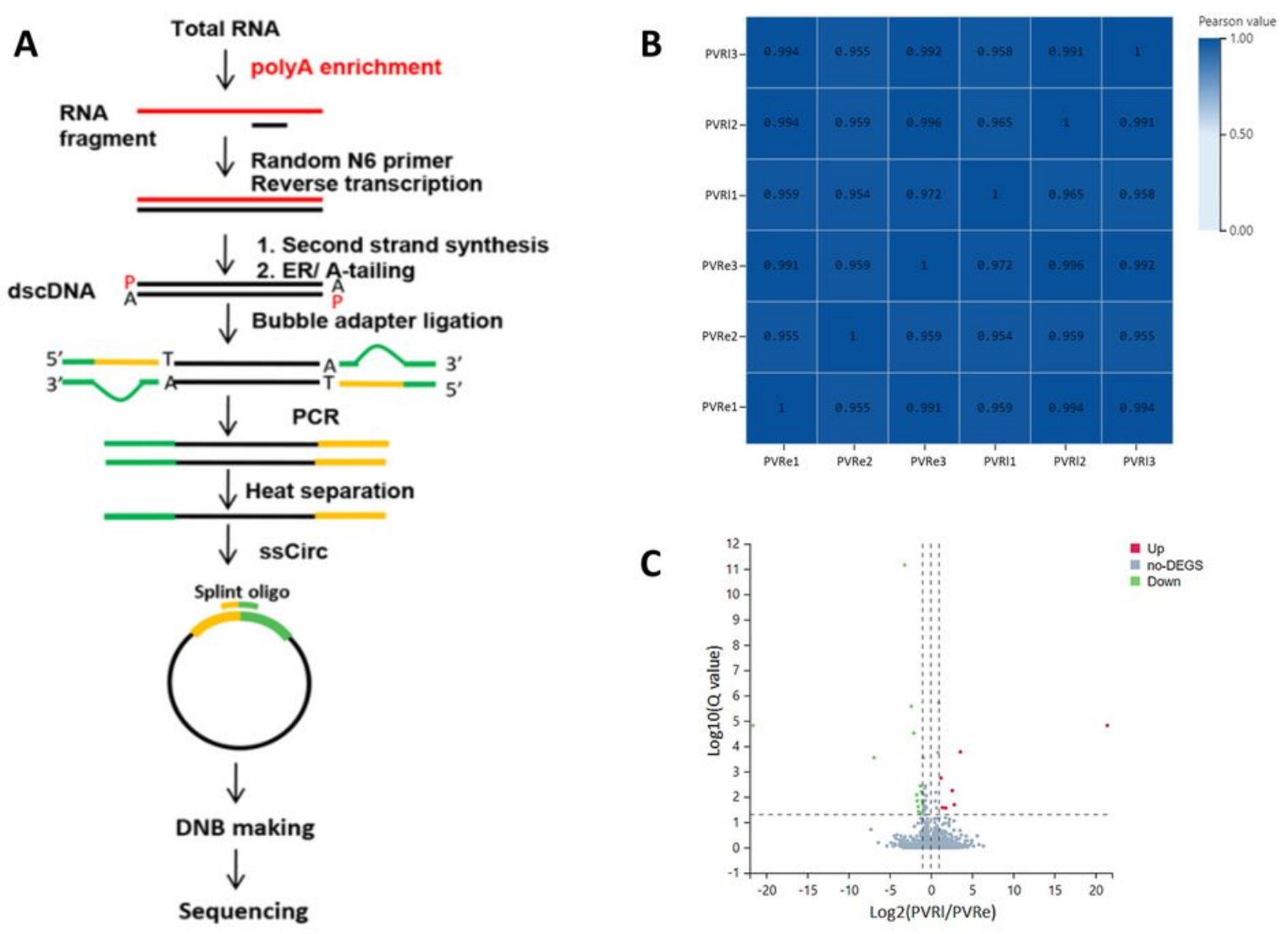

\section{Figure 1}

A Experimental flow chart of transcriptome sequencing. B Sample correlation heat map. (The $X$ and $Y$ axis represents each sample, and the color represents the correlation coefficient (the darker the color, the higher the correlation). C DGEs volcano plot. (The X-axis represents the log2 transformed fold change, and the Y-axis represents the -log10 transformed significance value. Red dots are up-regulated DEGs, blue dots are downregulated DEGs, and gray dots are non-DGEs). 

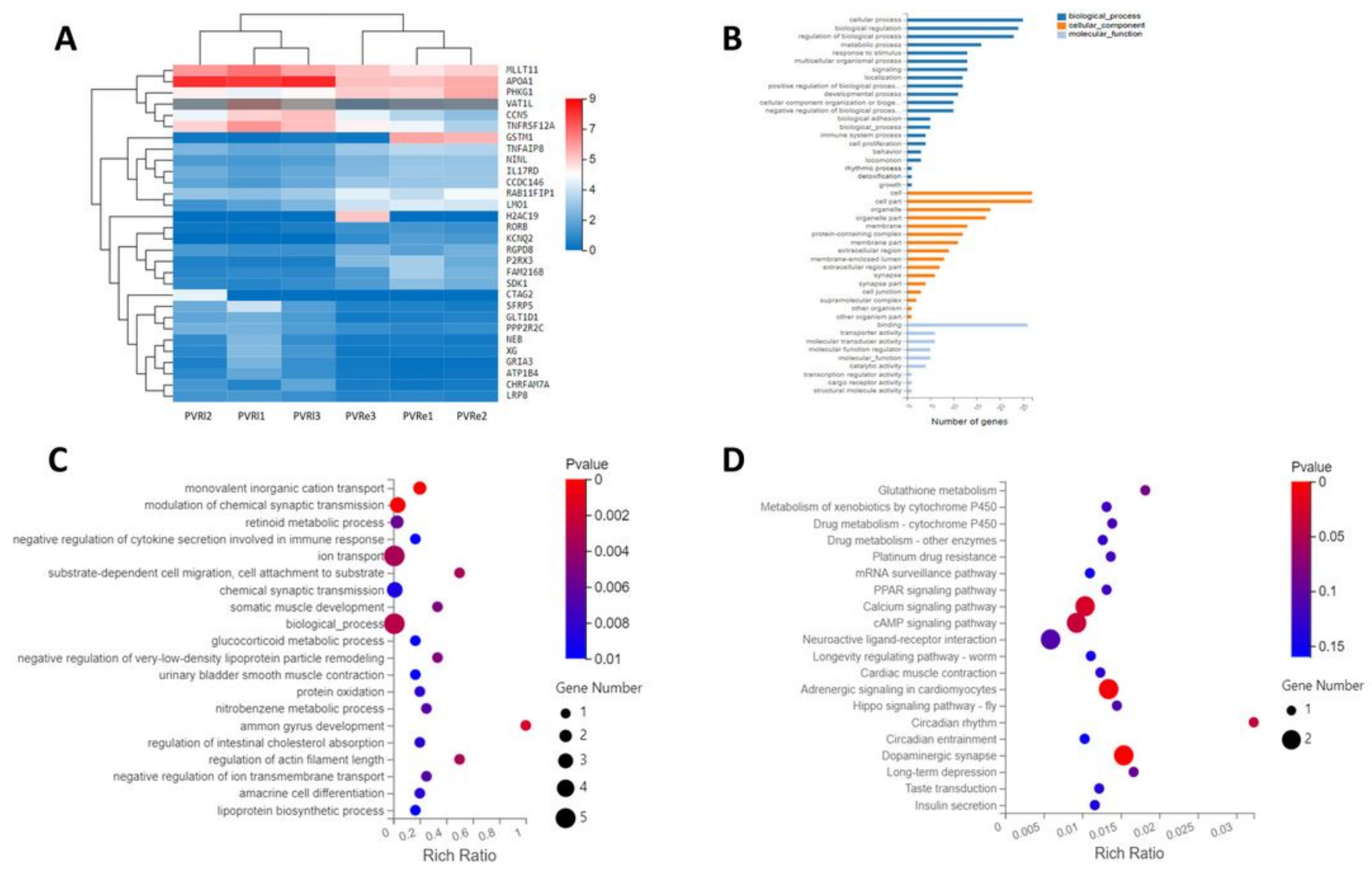

\section{Figure 2}

A Heatmap of hierarchical clustering of DEGs between PVRe compared to PVRI. (X axis represents each sample, $Y$ axis represents DEGs. Coloring indicates the log2 transformed fold change. High: red, Low: blue). B The GO classification results between PRle and PVRI. (X axis represents number of DEGs, Y axis represents GO terms). C GO biological processes enrichment of DEGs between PVRe and PVRI. D KEGG pathway functional enrichement of DGEds between PVRe and PVRI. 